\title{
Männlichkeit: Eine begriffliche Annäherung in phänomenologischer Perspektive
}

ÍNGRID VENDRELL FERRAN

\section{MÄnNLIChKEIT: StRATEgIEN ZUR Definition}

Anders als in vielen aktuellen Debatten in den Sozialwissenschaften, in denen die Themen „Männerstudien“ und „Männlichkeitsstudien“ seit den 80er Jahren viel Aufmerksamkeit erhalten haben und Gegenstand sorgfältiger Forschung gewesen sind, hat die Philosophie der letzten Jahrzehnte sich mit dem Begriff der Männlichkeit kaum befasst. ${ }^{1}$ Als akademische Disziplin scheint sie sich entweder für die Untersuchung dieses Phänomens nicht zu interessieren oder sie als die Aufgabe anderer Disziplinen zu verstehen. Die Klage der Soziologin Sylka Scholz, dass trotz der vielen Studien zur Männlichkeit das Konzept „erstaunlich wenig theoretisch fundiert [sei]“, gilt für die Philosophie in noch viel virulenterer Form: Die defizitäre Theoretisierung liegt allerdings nicht in einer mangelhaften Rezeption der verschiedenen zirkulierenden Konzepte von Männlichkeit und auch nicht in einem Streit über ihre Bedeutung, sondern in der Tatsache, dass das Phänomen nicht einmal als würdiges Untersuchungsobjekt betrachtet wird. ${ }^{2}$ Dieser

1 Ausschlaggebend in den Sozialwissenschaften war das 1995 veröffentlichte Buch von Raewyn Connell Masculinities, in dem die Autorin das Konzept der „hegemonialen Männlichkeit“ prägt (vgl. Connell, Raewyn: Masculinities, Berkeley, California/Los Angeles 2005). Im deutschsprachigen Raum wurde der Begriff vor allem von Michael Meuser untersucht. Vgl. für eine Darstellung der verschiedenen soziologischen Konzepte von Männlichkeit im deutschsprachigen Raum: Scholz, Sylka: Männlichkeitssoziologie, Münster 2015, S. $11 \mathrm{ff}$.

2 Für diese Diagnose einer Unterbestimmtheit in der Soziologie vgl. Scholz: Männlichkeitssoziologie, S. 11. 
Aufsatz hat als Ziel, ausgehend von philosophischen Positionen eine begriffliche Annäherung an dieses Konzept zu versuchen. Konkret geht es darum, aus einem phänomenologischen Hintergrund einen Beitrag zur theoretischen Fundierung dieses Begriffs zu leisten.

In vielen Diskursen in unserem Alltag wird darüber gesprochen, dass eine Person, eine Handlung, ein Gedanke, eine Gesellschaftsstruktur usw. „männlich“ sei. Auch wenn wir zunächst ein intuitives Verständnis davon haben können, was damit gemeint ist, scheint dieses Konzept sich einer genauen Untersuchung zu entziehen. Zum einem erweckt der Begriff den Eindruck, dass er mehr sagen will, als er auf den ersten Blick zu sagen scheint. Die Zuschreibung von „Männlichkeit" beschreibt nicht bloß einen Bereich der Realität, sondern ist mit einer Bewertung derselben verbunden. Wir haben es mit einem Begriff zu tun, der eher evaluativ als deskriptiv verwendet wird. Zum anderen besteht kein Konsens darüber, welche Eigenschaften genau der Kategorie „Männlichkeit“ zukommen. Die verschiedenen Kandidaten dafür, diese Kategorie näher zu bestimmen, lassen sich nicht immer miteinander versöhnen, sodass es kein einheitliches Bild davon gibt, was als ,männlich“ gilt oder gelten sollte. Der evaluative Charakter des Terminus und seine Mehrdeutigkeit bilden daher die zwei Hindernisse für eine Definition dieses Begriffs. ${ }^{3}$

Trotz der Schwierigkeiten benötigt dieses Konzept eine begriffliche Klärung, denn es handelt sich um einen weitverbreiteten und in unserer Sprache tief verankerten Terminus. Die Klärung soll in diesem Aufsatz mithilfe dreier philosophischer Strategien unternommen werden. Erstens werde ich zwei philosophische Modelle von Männlichkeit einander gegenüberstellen. Harvey C. Mansfields Theorie soll mithilfe von Pierre Bourdieus Konzept der männlichen Herrschaft kritisiert werden. Während Mansfield eine verbreitete Auffassung von Männlichkeit vertritt, die diese mit Assertivität, Mut und Adel verbindet, bietet Bourdieu uns ein gutes Instrumentarium dafür, versteckte Strukturen der männlichen Ordnung hinter diesem Bild zu entdecken. Zweitens wird diese Kritik mit Bourdieu durch einen phänomenologischen Hintergrund motiviert. Dabei werde ich sowohl die phänomenologischen Wurzeln von Bourdieus Denken als auch

3 Den Begriff der Weiblichkeit werde ich in diesem Aufsatz als komplementär zum Begriff der Männlichkeit verwenden und mit ihm eine Gruppe von Eigenschaften bezeichnen, die in einem bestimmen historischen Moment und sozialen Kontext Frauen zugeschrieben werden. Mein Vorschlag, Männlichkeit als offenen Begriff zu verstehen, lässt sich auch auf den Begriff der Weiblichkeit anwenden, auch wenn dabei andere Kriterien ins Spiel kommen. Der Fokus dieses Artikels auf Männlichkeit wird dadurch gerechtfertigt, dass es sich um ein bislang viel weniger analysiertes Konzept als Weiblichkeit handelt. 
die Möglichkeit der phänomenologischen Weiterführung seiner Ideen berücksichtigen. Besondere Aufmerksamkeit wird den Übereinstimmungen von Bourdieu und Max Scheler gewidmet. Schließlich werde ich den Vorschlag machen, „Männlichkeit“ als einen offenen Begriff zu verstehen, der historisch, sozial und kulturell wandelbar ist. Die Idee des offenen Begriffs bietet eine Alternative zu essenzialistischen Definitionen anhand notwendiger und hinreichenden Kriterien, lässt genügend Freiraum, um die Interdependenz zwischen gesellschaftlichem Wandel und unseren Männlichkeitskonstruktionen zu berücksichtigen und bleibt dem phänomenologischen Postulat treu, sich dem Phänomen in erster Instanz frei von theoretischen Konstrukten und ausgehend von Erfahrung anzunähern.

\section{MÄNNLICHKEIT ALS ASSERTIVITÄT UND MUT: MANSFIELDS THEORIE}

In diesem Abschnitt soll einer der wenigen aktuellen philosophischen Beiträge zu Männlichkeit dargestellt werden. Es handelt sich um die Monografie Manliness, die 2006 von Harvey C. Mansfield veröffentlicht wurde. Darin versucht der Autor eine bestimmte Auffassung dazu zu vertreten, was in den westlichen Gesellschaften lange als das Hauptbild von Männlichkeit galt und in jüngster Zeit durch die Geschlechterforschung stark infrage gestellt wurde. Diese Auffassung verbindet Männlichkeit mit bestimmten Charaktermerkmalen wie Aggressivität, Durchsetzungsvermögen und Mut.

Provokant ist dieses Buch in vielerlei Hinsichten. Der Autor vertritt eine sehr konservative Rollenaufteilung zwischen den Geschlechtern; er macht den Feminismus dafür verantwortlich, Frauen und Männer verwirrt und zu der Überzeugung gebracht zu haben, dass sie als Individuen genderneutral seien; er interpretiert Männlichkeit als eine Tugend und versucht für sie eine Funktion in der heutigen genderneutralen Gesellschaft zu finden. Diese kontroversen Thesen sind wahrscheinlich die Hauptgründe dafür, dass das Buch in Kontinentaleuropa bislang kaum rezipiert worden ist. Eine Analyse des Buches scheint mir dennoch aus verschiedenen Gründen sinnvoll. Es handelt sich um eine der wenigen philosophischen Veröffentlichungen zum Thema, die in jüngster Zeit verfasst wurden. Sie basiert auf den Auslegungen verschiedener Philosophen, die seit der Antike das westliche Denken stark geprägt haben. Die beschriebene Auffassung von Männlichkeit ist nicht nur in akademischen Zirkeln dominant, sondern besonders in vielen Diskursen unseres Alltagslebens. Die Tatsache, dass das Buch eines renommierten politischen Philosophen (Mansfield) von einer renommierten Uni- 
versität (Harvard) und in einem renommierten Verlag (Yale University Press) veröffentlicht wurde und unter seinen Kritikern auch renommierte Philosophinnen waren (Nussbaum), soll nicht aus den Augen geraten. Damit will ich nicht Autoritätsargumente dafür bringen, mich mit dem Buch zu befassen, sondern auf die Tatsache hinweisen, dass die darin vertretenen Thesen nicht obsolet sind, sondern die Meinung eines Teils unserer Gesellschaft widerspiegeln.

Männlichkeit wird im Buch als hervorragende Eigenschaft oder Tugend verstanden, die hauptsächlich bei Männern zu finden sei, aber auch bei Frauen beobachtet werden könne (als Beispiel einer männlichen Frau nennt Mansfield Margaret Thatcher). ${ }^{4}$ Der Autor geht davon aus, dass es Männer und Frauen als biologisch unterschiedliche Kategorien gibt und Männer dazu neigen, mehr Aggressivität als Frauen zu zeigen. Diese Beobachtung entwickelt Mansfield mithilfe philosophischer Auffassungen über Männlichkeit weiter, die er bei Plato, Aristoteles, den Stoikern, Machiavelli, Rousseau, Nietzsche u.a. findet. Daraus entwickelt er eine nicht eindeutig bestimmte Liste von Merkmalen, die sowohl positive als auch negative Eigenschaften bezeichnen. Nicht klar ist, ob alle oder nur einige diese Eigenschaften und bis zu welchem Grad diese vorhanden sein sollen, damit etwas als „männlich“ bezeichnet werden kann. Dennoch geben zwei Eigenschaften den Grundton seiner Untersuchung vor. Zum einem gibt es die „Assertivität", die er als eine Fähigkeit versteht, entschlossen zu sein, ohne dass diese Entschlossenheit unbedingt von einer Kenntnis der Sachlage begleitet würde. Zum anderen erwähnt er den „Mut“, den er anhand des antiken Begriffs des „Thymos“ erklärt. So schreibt Mansfield: „Thumos is a quality of spiritedness, shared by humans and animals, that induces humans, and especially manly men, to risk their lives in order to save their lives. “5 Mit dem Mut ist auch die Angst des männlichen Mannes verbunden, diese Eigenschaft zu verlieren, sodass beide Emotionen als zwei Seiten einer Medaille verstanden werden sollen. Auch weitere Aspekte wie etwa Selbstvertrauen - oder, wie Mansfield es ausdrückt: „[c]onfidence in the face of risk ${ }^{\text {“6 }}$-, Verteidigung des Eigenen, Aggression und Überdramatisierung sind für „Männlichkeit“ kennzeichnend.

Dieses Bild von Männlichkeit aber - so Mansfields Feststellung - steht in unserer heutigen Gesellschaft unter Spannung. Einerseits wirkt es auf uns noch attraktiv, weil wir mit dieser Eigenschaft Selbstvertrauen und die Fähigkeit zu kommandieren assoziieren. ${ }^{7}$ Andererseits aber wird in der heutigen genderneutralen Gesellschaft „Männlichkeit“ als Stereotyp angesehen. Um dieser Spannung zu ent-

4 Mansfield, Harvey C.: Manliness, Yale 2006, S. XII.

5 Ebd., S. XI.

6 Ebd., S. 23.

7 Vgl. ebd., S. 16. 
kommen, versucht Mansfield für dieses Bild von Männlichkeit in der heutigen Gesellschaft eine Funktion zu finden. Zunächst hält er die Stereotype für richtig, denen zufolge Männer aktiv, aggressiv usw. und Frauen passiv und gesellig seien. ${ }^{8}$ Sie funktionieren als notwendige Kategorien, um die Realität zu ordnen, und diese Zuschreibungen haben alle Revolutionen außer der Frauenbewegung überlebt. Im Folgenden geht er von einer Opposition zwischen „Männlichkeit“ einerseits und „rationaler Kontrolle“ der Moderne andererseits aus, die mit Assertivität und Mut den Hauptmerkmalen der Männlichkeit - wenig zu tun hat, weil die Moderne auf Professionalität und Sicherheit basiert. Da die heutige Gesellschaft alles unter Kontrolle haben will und sich als genderneutral versteht, ist die Kategorie der Männlichkeit - so Mansfield - „,arbeitslos“ oder „,ohne Beschäftigung“ (,unemployed"). ${ }^{9}$ Demokratie und auch Feminismus versteht er als Implementierungen dieser Idee der rationalen Kontrolle. Eben darin liegt Unangemessenheit der feministischen Strömung - so seine These -, denn sie sei richtig hinsichtlich der Idee, dass Männlichkeit teilweise sozial konstruiert ist, aber falsch in der Annahme, dass Männer gegen ihre Neigungen transformiert werden könnten. Seinen Vorschlag, wie Männlichkeit wieder zu ,,beschäftigen“ ist, fasst er wie folgt zusammen:

„Our gender-neutral society needs to readopt the distinction between public and private that is characteristic of liberalism. In public it should be gender-neutral, in private not. In public it should not permit sex stereotypes to operate; in private it should admit that they are true. True means true as generalities, not in every case. “10

Er ist der Meinung, dass wir die Bürger als genderneutral, die Menschen in der privaten Sphäre jedoch als geschlechtlich eingeteilt (,gendered“) denken sollten. Die Individuen in einer Gesellschaft sollten leben, als ob sie verheiratet wären: ,[T]he men protective and authoritative as if they were husbands, the women nurturing and critical as if they were wives. "11 Frauen hätten in der genderneutralen Gesellschaft die Funktion zu kontrollieren, dass keine Situationen eintreten, in denen es ,zu viel“ Männlichkeit gibt (d.h. zu viel Aggression usw.). ${ }^{12}$

8 Vgl. ebd., S. 32. Auch wenn Mansfield viele der Stereotype über Männer und Frauen für richtig hält, arbeitet er nicht mit den typischen Klischees, die besagen, dass Frauen überemotional und irrational seien.

9 Ebd., S. 229.

10 Ebd., S. 241.

11 Ebd., S. 242.

12 Vgl. für eine Kritik dieser These: Titunik, Regina: Undermanned, in: H-Minerva, April 2007, S .1-3 hier S. 1, unter: https://networks.h-net.org/node/5376/reviews/37050/ titunik-mansfield-manliness 
Unreflektiert bleiben in Mansfields Werk dennoch die verschiedenen Ideologien, die hinter dem Bild von Männlichkeit stecken, das er vertritt. So geht er zum Beispiel von der Idee einer Genderneutralität unserer Gesellschaft aus. Dabei ignoriert er, dass trotz vieler Gesetze, die eine Gleichberechtigung zu garantieren versuchen, die Individuen in einer Gesellschaft mit Wahrnehmungs- und Denkmustern operieren, die nicht genderneutral sind. Historisch betrachtet haben Frauen und Männer erst seit kurzem denselben rechtlichen Status. Die Mentalitäten, Einstellungen, Erwartungen und Werte der Individuen und der Gesellschaft ändern sich nur allmählich und unterliegen viel langsameren sozialen Wandlungsprozessen als die Formulierung eines neuen Gesetzes. ${ }^{13}$ Auch Mansfields Bild des Feminismus ist stark ideologisch geprägt, da er davon ausgeht, dass Feministen die Geschlechterunterschiede in der öffentlichen und privaten Sphäre abschaffen wollten. Wie Nussbaum aber zutreffend kritisiert hat, zielt der Feminismus nicht auf eine Gesellschaft ohne Differenzierung der Geschlechter ab, sondern auf eine Kritik der Gesellschaft, die die Rechte und Freiheiten der Menschen beschränkt. ${ }^{14}$ Feminismus strebt eine Gesellschaft ohne Geschlechterhierarchien an, und diese Gesellschaft ist heutzutage nicht vorhanden. Auch die Annahme, dass es eine biologische Tatsache sei, dass es Männer und Frauen gebe und diesen zwei Kategorien unterschiedliche Charaktermerkmale zugeschrieben werden können, übernimmt er unkritisch, ohne den Einfluss der sozialen Umgebung zu berücksichtigen, uns Klassifikationen zu diktieren,. Schließlich lässt er auch die Assoziation zwischen Männlichkeit, Mut und Tugend unhinterfragt, die er für selbstverständlich erachtet. Kurzum: Der Beitrag ignoriert, dass über Männlichkeit zu sprechen eine Reflexion über das Verhältnis zwischen den Geschlechtern und den Einfluss der sozialen Ordnungen, in denen wir leben, impliziert. Eben diese Reflexion über Machtverhältnisse ist zentral für Bourdieus Analysen der männlichen Herrschaft, die im Folgenden in phänomenologischer Perspektive zu entwickeln sind.

13 Sztompka, Piotr: The Sociology of Social Change, Oxford, UK/Cambridge, Massachusetts 1993.

14 Nussbaum, Martha C.: „Man Overboard“, in: The New Republic vom 28.6.2006, S. 31f. 


\section{Bourdieu Und die Phänomenologie}

\subsection{Die unbewusste Verinnerlichung historischer Strukturen der männlichen Ordnung}

In folgenden Abschnitten soll ein Bild von Männlichkeit dargestellt und weiterentwickelt werden, das der immer noch herrschenden Auffassung, die Mansfield in seinem Buch exemplarisch illustriert, kritisch gegenübersteht. Als Ausgangspunkt wird das 1998 veröffentlichte Buch von Pierre Bourdieu Die männliche Herrschaft dienen. ${ }^{15}$ Auch wenn dieser sich auf eine ethnografische Untersuchung der Berber der Kabylei stützt, bieten seine Analysen ein gutes Werkzeug dafür, die heute noch herrschende Auffassung von Männlichkeit zu kritisieren, die oben erörtert wurde. Die phänomenologische Perspektive wird einbezogen, indem die Übereinstimmungen des Denkens des Autors mit dieser philosophischen Tradition aufgedeckt und seine Thesen mithilfe phänomenologischer Überlegungen weitergeführt werden.

Bourdieu macht in seinem Text darauf aufmerksam, dass das Denken über Männlichkeit immer in den Parametern der verinnerlichten Strukturen der sozialen Ordnung stattfindet. So schreibt er, dass Menschen ,in Form unbewusster Wahrnehmungs- und Bewertungsschemata die historischen Strukturen der männlichen Ordnung verinnerlicht" haben. ${ }^{16}$ Damit warnt er uns, dass wir, indem wir über Männlichkeit (und auch über die männliche Herrschaft) nachdenken, an Strukturen appellieren, die selbst ein Ergebnis dieser Herrschaft sind. Jeder Akt der Erkenntnis ist - so Bourdieu - daher auch ein Akt der Anerkennung eines alten Paradigmas. Die männliche Herrschaft reproduziert sich anhand zweier Mechanismen. Zum einen werden soziale Konstruktionen naturalisiert: Eine bestimmte Klassifikation und Ordnung der Welt, die gesellschaftlich konstruiert wurde, scheint uns in der Natur der Dinge selbst zu liegen. Dieses Phänomen bezeichnet er als „Paradox der Doxa“"17. Als Beispiel dient ihm die Einteilung der Welt in männlich und weiblich in der kabylischen Gesellschaft, die Ergebnis einer Konstruktion ist und den Berbern als natürlich erscheint. Zum anderen wird die männliche Herrschaft im Habitus der Individuen inkorporiert. Dies geschieht,

15 Vgl. für eine Auslegung und Kritik von Bourdieus Hauptbegriffen und besonders des Habitusbegriffs: Guthoff, Heike: Kritik des Habitus. Zur Intersektion von Kollektivität und Geschlecht in der akademischen Philosophie, Bielefeld 2013, S. 47ff.

16 Bourdieu, Pierre: Die männliche Herrschaft, übersetzt v. Jürgen Bolder, Frankfurt a.M. 2005, S. 14.

17 Ebd., S. 7. 
indem ein konkretes Wahrnehmungsschema von den Individuen habitualisiert wird.

Diese Ideen Bourdieus haben einen starken phänomenologischen Hintergrund und können auch im Rahmen dieser Denkströmung weiterentwickelt werden. So scheint es starke Übereinstimmungen mit Schelers Ideen über unsere Formen des Weltbezugs zu geben. Scheler analysiert in seinem Werk die „Erwartungsschemata“ - manchmal auch metaphorisch als „Fenster“, „Herz“ oder „Ordo Amoris“ bezeichnet-, die die Formen des Weltbezugs von Individuen und Kollektiven charakterisieren. Damit ist eine grundlegende intentionale Beziehung gemeint, mit der sich Individuen und Kollektive auf die Realität beziehen. Diese Grundhaltung ist nicht neutral und in ihr sind bestimmte Richtungen unseres Weltbezugs zu erkennen: was uns interessiert, die Eigenschaften oder Werte, die in den Vordergrund treten sowie die Präferenzen unseres Weltzugangs. Jeder Mensch und jede Epoche, Kultur, Gesellschaft usw. wird - so Scheler - durch eine bestimmte und für sie charakteristische Form des Weltbezuges bestimmt.

Ein erster interessanter Aspekt dieser These ist, dass das primäre Verhältnis des Menschen zur Welt nie rein kontemplativ oder theoretisch, sondern immer von Interessen und Motiven bedingt ist. ${ }^{18}$ Diese Bedingtheit versteht Scheler als eine affektive Form des Weltbezugs. Das heißt, dass unser Wahrnehmen, Denken und Wollen immer ausgehend von einer Situation stattfindet, die uns affektiv betrifft. Diese primäre affektive Form des Weltbezugs bestimmt, was in unser Wahrnehmungsfeld fällt, was wir über die Realität denken und wie wir uns im Handeln orientieren. So schreibt er: „Der Mensch ist, ehe er ein ens cogitans ist oder ein ens volens, ein ens amans. ${ }^{19}$ Dieses Betroffensein soll nicht im Sinne eines konkreten Gefühls interpretiert werden, sondern im Sinne einer grundlegenden Affektivität, die Wahrnehmen, Denken und Wollen bestimmt. Im Vergleich zu dieser ursprünglichen Form von Affektivität sind die Gefühle in der Phänomenologie Max Schelers komplexe Phänomene, die auf Kognitionen wie Wahrnehmungen oder Urteilen fußen.

Ein weiterer Aspekt dieser These ist der folgende: Auf der individuellen Ebene besagt sie, dass jeder Mensch sich durch eine einzigartige Form des Weltbezugs auszeichnet, von der seine Interessen, seine Aufmerksamkeit und sein Erkennen und Wollen abhängig sind. Auf der gesellschaftlichen Ebene bedeutet

18 Er spricht in diesem Zusammenhang von der praktischen Bedingtheit der Wahrnehmung. Vgl. Scheler, Max: Erkenntnis und Arbeit, in: ders., Gesammelte Werke, Band VIII, hrsg. v. Maria Scheler, Bern 1960, S. 191-382, hier S. 239 und 361f.

19 Scheler, Max: Ordo Amoris, in: ders., Gesammelte Werke, Band X, hrsg. v. Manfred S. Frings, Bonn 1986, S. 345-376, hier S. 356 [Hervorh. i. Orig.]. 
sie, dass auch jedes Kollektiv seine Formen des Weltbezugs, seine Rangordnungen und Präferenzen hat. Da wir als Individuen immer in verschiedene kollektive Einheiten (Familie, Gemeinschaft, Kultur usw.) eingebettet sind, sind wir von diesen anderen Formen, sich auf die Welt zu richten, stark beeinflusst und mitbestimmt. Wir richten uns daher immer im Rahmen dieser Möglichkeiten auf die Welt. Diese verschiedenen Formen des Weltbezugs stehen somit in einem $\mathrm{Zu}$ sammenspiel und jeder von uns kann seinen Weltbezug nur im Rahmen dieses Zusammenspiels bestimmen.

Schließlich ist in diesem Kontext Schelers These wichtig, dass es nicht nur einen Einfluss der kollektiven Formen auf jede individuelle Form des Weltbezugs gibt, sondern auch umgekehrt. Es ist möglich, dass bestimmte Individuen einen Einfluss auf die kollektive Ebene ausüben. Dies geschieht etwa, wenn ein bestimmtes Individuum für einen Aspekt der Realität empfindlich wird, der vorher keine Aufmerksamkeit erhielt, verborgen blieb oder nicht in das herrschende Präferenzsystem passte und die anderen dafür sensibilisiert.

Diese Ideen sind aufschlussreich, um die Interdependenz zwischen Individuum und Gesellschaft zu verstehen und Bourdieus These einer „Verinnerlichung“ näher zu erläutern. Es handelt sich nicht um einen Einfluss des Äußeren auf das Innere, sondern um die Tatsache, dass wir als Menschen in mehrere Kollektive eingebettet sind, die unsere individuelle Form des Weltbezugs mitbestimmen. ${ }^{20}$ Sie bieten uns ein mögliches Erklärungsmuster dafür, inwiefern der soziale Wandel in einer Gesellschaft bestimmte Auffassungen der Realität verändern kann. Konkret für den Fall des Begriffs von „Männlichkeit“ zeigt dies, inwiefern sich, wenn wir über Männlichkeit nachdenken, unsere Wahrnehmungs-, Denk- und Handlungsmuster im Rahmen der Paradigmen der verschiedenen Kollektive bewegen, in denen wir leben. Möglich ist dann, dass wir mit Strukturen der männlichen Ordnung operieren, die zu einem bestimmten Paradigma von Männlichkeit gehören, das die Geschlechter nicht als gleichberechtigt betrachtet.

Darüber hinaus kann es auch der Fall sein, dass dieses spezifische Männlichkeitsbild andere mögliche Formen von Männlichkeit, die nicht zu ihm passen, als untergeordnet betrachtet. Dieser Aspekt wurde etwa von Scholz bemerkt. Sie hat hierzu vorgeschlagen, Bourdieus Konzept der männlichen Herrschaft zu erweitern, und bedient sich dafür der Idee einer hegemonialen Männlichkeit bei Connell, der zufolge es verschiedene Konstruktionen von Männlichkeit gibt und die-

20 Wie Scholz kritisiert hat, wird oft von einem Einfluss des Äußeren auf das Innere gesprochen, ohne dass erklärt wird, wie dieses Zusammenspiel stattfindet. Vgl. Scholz: Männlichkeitssoziologie, S. 20. 
se hierarchisch strukturiertsind. ${ }^{21}$ Diese Idee ist wichtig, weil sie es erlaubt, Machtbeziehungen nicht nur zwischen Männern und Frauen, sondern auch von Männern untereinander zu verstehen. Scholz nimmt den Begriff der männlichen Herrschaft als einen exemplarischen Fall von symbolischer Herrschaft und versucht, verschiedene Herrschaftsphänomene zu berücksichtigen. ${ }^{22}$ Dies impliziert, nicht nur die Überordnung von Männlichkeit über Weiblichkeit, sondern auch einer hegemonialen Männlichkeit über andere Formen von Männlichkeit zu berücksichtigen.

\subsection{Die soziale Bedingtheit von Emotionen und emotionaler Beziehungen}

Verbreiteter Teil des Bildes von Männlichkeit, das Mansfields Beitrag exemplarisch verkörpert und das sich aus dem Gesichtspunkt Bourdieus und der Phänomenologie kritisieren lässt, ist die These, dass jedem Geschlecht bestimmte Merkmale zu eigen sind. Diesem Bild zufolge wird einerseits zwischen Männern und Frauen als biologischen Kategorien differenziert und andererseits werden das Männliche und das Weibliche als Eigenschaften unterschieden, die jeweils „eher“ an Männern oder Frauen vorhanden sind.

Alternativ zu diesem Bild zeigt Bourdieu in seinem Buch, inwiefern die Einteilung der Geschlechter eine gesellschaftliche und nicht eine „biologische“ Tatsache ist. Seine Überlegungen zeigen, dass die biologischen Notwendigkeiten der Reproduktion nicht die Organisation der Geschlechter und die soziale Ordnung, die daraus entsteht, rechtfertigen. Jedes Geschlecht ist das Produkt einer Konstruktionsarbeit und die biologischen Unterschiede können die sozialen Unterschiede nicht legitimieren. Somit handelt es sich um eine Umkehrung der Tatsachen, wenn der gesellschaftlich konstruierte Unterschied uns als das Natürliche erscheint. ${ }^{23}$ Ferner ist die Verwechslung von Männlichkeit und männlichem

21 Ebd., S. 23 und 26. Scholz versteht die Idee einer hegemonialen Männlichkeit als institutionalisierte Praxis. Dies erlaubt ihr eine Rekonstruktion ihrer historischen Formen. Die Hauptideen des Konzeptes -Vielfalt und Hierarchie von Männlichkeiten bleiben damit erhalten. Vgl. Connell, Raewyn/Messerschmidt, James W.: Hegemonic Masculinity. Rethinking the Concept, in: Gender \& Society 19/6 (2005), S. 829-859, hier S. 846.

22 Vgl. Scholz: Männlichkeitssoziologie, S. 23 und 31.

23 Vgl. Bourdieu: Die männliche Herrschaft, S. 23. 
Geschlecht, die viele Bilder von Männlichkeit charakterisiert, Symptom eines naturalistischen Fehlschlusses. ${ }^{24}$

Die genannte Konstruktionsarbeit betrifft nicht nur die Einteilung der Geschlechter, sondern auch die Merkmale, die jedem Geschlecht zugeschrieben werden. Es gibt einen sehr aufwändigen Prozess der Maskulinisierung des männlichen und der Feminisierung des weiblichen Körpers. Der Körper, so Bourdieu, wird durch die Wirkungen mimetischer Suggestionen transformiert, durch explizite Einschärfung und die symbolische Konstruktion der Sicht des biologischen Körpers. ${ }^{25}$ Dies lässt sich am besten am Beispiel von Gefühlen und Emotionen illustrieren. Bourdieu spricht von Liebe, Bewunderung und Respekt, aber auch von Scham, Erniedrigung, Schüchternheit, Beklemmung, Ängstlichkeit, Zorn und ohnmächtiger Wut, die sich in äußeren Anzeichen wie Erröten, Zittern usw. ausdrücken. ${ }^{26}$ Diese Emotionen, die als sehr individuell, persönlich, subjektiv und natürlich erfahren werden, verkörpern in Wirklichkeit Akte des Erkennens und Anerkennens zwischen Beherrschten und Herrschenden. Sie können nicht von den Strukturen getrennt werden, in denen sie stattfinden. Viele der Gefühle, die mit Männlichkeit in Verbindung gebracht werden, sind nicht angeboren, sondern sozial verinnerlicht. ${ }^{27}$ Assertivität und Mut, die den Kern des Bildes von Männlichkeit darstellen, das im vorigen Abschnitt beschrieben wurde, sind daher keine Kategorien, die Männlichkeit wesentlich definieren, sondern Produkt einer Konstruktionsarbeit.

Auch diese Idee findet im Rahmen der Phänomenologie Platz. Frühe Phänomenologen wie Scheler und José Ortega y Gasset oder im Rahmen der jüngsten Entwicklungen dieser Strömung Hilge Landweer haben dafür plädiert, einen Zusammenhang zwischen unseren Gefühlen und den sozialen Strukturen, in denen wir leben, zu sehen. ${ }^{28}$ Der Einfluss der Gesellschaft auf emotionale Dispositionen, Veranlagungen, Emotionen und Stimmungen drückt sich auf verschiedenen

24 Guthoff erwähnt einen ähnlichen Fehlschluss bei Connell. Vgl. Guthoff: Kritik des Habitus, S. 11.

25 Vgl. Bourdieu: Die männliche Herrschaft, S. 99.

26 Vgl. ebd., S. 72.

27 Vgl. ebd., S. $94 f$.

28 In diesem Sinne sind die Analysen Schelers zu Scham und Schamgefühlen, Ortega y Gassets zur Liebe sowie Landweers zur Echtheit von Gefühlen aufschlussreich. Vgl. Scheler, Max: Über Scham und Schamgefühl, in: ders., Gesammelte Werke, Band X, hrsg. v. Maria Scheler, Bern 1957, S. 67-154; Ortega y Gasset, José: Über die Liebe. Meditationen, übersetzt v. Helene Weyl, Stuttgart 1957; Landweer, Hilge: Sozialität und Echtheit der Gefühle. Geschlechtertheoretische Perspektiven, in: Agnes Neumayr (Hg.), Kritik der Gefühle, Wien 2007, S. 63-91. 
Ebenen aus. Sozial bedingt sind viele der Ausdrucksformen, die unsere Gefühle annehmen können sowie die Intensität, die als berechtigt für ihren Ausdruck gilt. Auch viele der Gegenstände, auf die sich unsere Gefühle richten und Situationen, die nach einer bestimmten emotionalen Reaktion verlangen, werden durch Sozialisation mitbedingt. In einigen Fällen ist es sogar möglich, dass die Existenz eines Gefühls von einer bestimmten Gesellschaft oder Kultur abhängig ist. Dabei denke ich etwa an die „höfische Liebe“, die heute nicht mehr vorhanden ist oder an die historische Entstehung der Idee der „romantischen Liebe“.

Es wäre m.E. ein Fehler, diese soziale Bedingtheit im Sinne eines radikalen Sozialkonstruktivismus zu interpretieren, wie dies bei manchen Soziologinnen und Soziologen geschehen ist. ${ }^{29}$ Die heutige Debatte bewegt sich zwischen den Alternativen ,angeboren“ und „sozial konstruiert“. Im ersten Fall hätten wir es mit einer Gruppe von Gefühlen zu tun - den „,basic emotions“-, die bei allen Menschen vorhanden wären. Diese Ansicht trifft sich mit Darwins Interpretation der Emotionen als universell in dem Sinne, dass sie ein Produkt der Evolution sind und wird heute von Autoren wie Ekman weiter vertreten. ${ }^{30}$ Im zweiten Fall würden Gefühle von den menschlichen Praktiken einer Gesellschaft erzeugt, in der sie auftreten. In diesem Zusammenhang bietet die Phänomenologie einen dritten Weg, um die soziale Bedingtheit der Gefühle zu verstehen. Die Phänomenologie, besonders so, wie sie Scheler verstanden hat, geht von einer ,universellen Grammatik“ ${ }^{31}$ der Gefühle aus. „Universell“ meint hier nicht, wie in der oben genannten Theorie der der „,basic emotions“, dass alle Menschen aus biologischen Gründen dieselben Grundemotionen besäßen, sondern bezieht sich auf eine allen Menschen gemeinsame Struktureinheit, die jeder Mensch qua Menschsein besitzt. Diese menschliche Grundstruktur ist dafür verantwortlich, dass wir die Emotionen anderer Menschen verstehen können, ohne sie zuvor selbst erlebt zu haben. Sie lässt allerdings genug Freiraum für individuelle und kollektive Variationen, was Ausdruck, Intensität, Gegenstände usw. der Emotionen betrifft. Diese Variationen sind ,angelernt“, sodass die universellen Aspekte unserer Gefühle durch Gesellschaft und Kultur modelliert werden. ${ }^{32}$

29 Ein Beispiel dafür ist Scholz: Männlichkeitssoziologie, S. 198ff.

30 Vgl. Ekman, Paul: Emotions Revealed. Recognizing Faces and Feelings to Improve Communication and Emotional Life, New York 2004.

31 Scheler, Max: Wesen und Formen der Sympathie, in: ders., Gesammelte Werke, Band VII, hrsg. v. Manfred S. Frings, Bern/München 1973, S. 9-258, hier S. 21.

32 Vgl. Ortega y Gasset, José: Über den Ausdruck als kosmisches Phänomen, in: ders., Gesammelte Werke, Band 1, übersetzt v. Helene Weyl und Ulrich Weber, Stuttgart 1954, S. 393-415, hier S. 405. 
Diese Überlegungen sprechen dafür, dass affektive Phänomene sozial bedingt sind. In diesem Kontext ist zu erwarten, dass es geschlechterspezifische Differenzen bei der Sozialisation in diesen verschiedenen Aspekten gibt. ${ }^{33}$ Dies erklärt, warum einige Charaktermerkmale, die als angeboren für ein Geschlecht erscheinen, in Wirklichkeit Resultat einer Konstruktionsarbeit sind (im Sinne einer Modellierung).

In diesen Zusammenhang ist auch die folgende Beobachtung einzuordnen: In westlichen Gesellschaften besteht eine Verbindung zwischen Weiblichkeit und Emotionalität einerseits und Männlichkeit und Rationalität andererseits. Nur bestimmte Emotionen werden in diesem Rahmen für Männer reserviert. Während Einfühlung, Fürsorge, romantische Liebe u.Ä. als weiblich betrachtet werden, werden Gefühle wie Aggression, Zorn, Mut, Durchsetzungsstärke usw. Männern zugeschrieben. ${ }^{34}$ Das in Abschnitt 2 dargestellte Bild von Männlichkeit steht im Rahmen dieser Tradition. Dieses Bild schreibt Frauen sanfte Gefühle zu (die Mansfield auch mit der rationalen Kontrolle der Moderne assoziiert). Männer werden dagegen entemotionalisiert, wenn es auch noch Freiraum für „starke Emotionen“"wie Wut oder Mut gibt. Diese Assoziation ist Produkt einer Naturalisierung sozialer Bedingtheiten.

Vor diesem Hintergrund lässt sich auch Mansfields Vorschlag kritisieren, demzufolge die Unterschiede zwischen dem Männlichen und dem Weiblichen in der Privatsphäre lebendig gehalten werden sollten. Er verlangt eine auf der öffentlichen Ebene genderneutrale Gesellschaft, aber die Spaltung der Rollen auf privater Ebene. Mansfield hält es für natürliche Tendenzen, dass Männer eher aggressiv und assertiv, Frauen hingegen eher pflegend und kooperativ seien; dies verwendet er als Legitimation dafür, dass das hierarchische Verhältnis der Geschlechter in der privaten Sphäre beibehalten wird (und nicht nur zwischen den Geschlechtern, sondern auch unter Individuen desselben Geschlechts, denn dies betrifft auch andere Formen von Männlichkeit, die nicht dieser dominanten Form entsprechen), weil derjenige, der Assertivität und Aggressivität zeigt, sich leichter in eine übergeordnete Position bringen kann, als diejenige, die für andere sorgt.

33 Vgl. Landweer: Sozialität und Echtheit der Gefühle, S. 63-91.

34 Vgl. Newmark, Catherine: Weibliches Leiden - männliche Leidenschaften. Zum Geschlecht in älteren Affektenlehren, in: Feministische Studien 26/1 (2008), S. 7-18 und dies.: Vernünftige Gefühle? Männliche Rationalität und Emotionalität von der frühneuzeitlichen Moralphilosophie bis zum bürgerlichen Zeitalter, in: Manuel Borutta/Nina Verheyen (Hg.), Die Präsenz der Gefühle. Männlichkeit und Emotion in der Moderne, Bielefeld 2010, S. 41-55. 


\subsection{Die ,vererbte‘ Assoziation von Männlichkeit und Tugend}

Ein dritter wichtiger Aspekt von Bourdieus Untersuchungen besteht darin, die Assoziation zwischen Männlichkeit und Tugend bzw. Virilität und Adel aufzudecken. Diese Assoziation wird durch eine Verbindung zwischen Männlichkeit und Mut - im Sinne von „Thymos“ - einerseits und Mut und Tugend andererseits legitimiert. Auch hinter dieser Assoziation steht ein hierarchisches Verhältnis zwischen den Geschlechtern, demzufolge das Männliche, als Adel verstanden, dem Weiblichen übergeordnet wird; diese Rangordnung betrifft dann auch die verschiedenen Verständnisse von Männlichkeit, da ein bestimmtes Bild anderen möglichen Bildern von Männlichkeit vorgezogen wird.

Eine solche Verbindung wird von Bourdieu in seinen ethnografischen Studien aufgewiesen. Bourdieu beobachtet, dass in der Gesellschaft der Berber genauso wie in der westlichen Tradition bis heute eine Verbindung zwischen Virilität, moralischem Mut und Adel besteht. So schreibt er: „Die Virilität bleibt, gerade auch unter ihrem ethischen Aspekt, d.h. als Quiddität des vir, virtus point d'honneur (nif), Prinzip der Wahrung und Mehrung der Ehre, zumindest stillschweigend mit der physischen Virilität verknüpft, und zwar insbesondere durch die vom wahren Mann erwarteten Beweise der Sexualkraft““. ${ }^{35}$ Diese Assoziation steht auch hinter Mansfields Theorie der Männlichkeit als Tugend, die er anhand philosophischer Texte seit der Antike zu stützen versucht. Die Tatsache, dass er sich auf eine lange philosophische Tradition stützt, zeigt, wie stark diese These in unserer westlichen Kultur verankert ist.

Diese Assoziation führt dazu, dass ein Mann zu sein auch impliziert, dass eine bestimmte Denk- und Handlungsweise erwartet wird, d.h., dass von etwas, das den Schein des Natürlichen hat, ein Seinsollen bzw. eine Tugend abgeleitet wird. ${ }^{36}$ Die Struktur der männlichen Herrschaft bestimmt daher auch, wie die Männer gesellschaftlich konditioniert sind. Sie müssen sich einem bestimmten Bild des Mannes stellen, einer ,illusio“, die darin besteht, andere Männer nach bestimmten Regeln des Spiels zu dominieren. ${ }^{37}$ Frauen werden von diesem Spiel ausgeschlossen. Zutreffend beobachtet Bourdieu, dass wir es hier nicht mit einer natürlichen Assoziation, sondern mit einer sozialen Konstruktion zu tun haben: „Der Adel oder der point d'honneur im Sinne einer Gesamtheit von Dispositionen, die als edel gelten (körperlicher und geistiger Mut, Großzügigkeit, Weitherzigkeit usf.), ist das Produkt einer sozialen Benennungs- und Einprägungsar-

35 Bourdieu: Die männliche Herrschaft, S. 23 und 25. Im selben Sinne: S. 106.

36 Vgl. ebd., S. $90 f$.

37 Vgl.ebd., S. 132f. 
beit"“ ${ }^{38}$ In phänomenologischen Termini kann gesagt werden, dass es sich um eine historisch, kulturell und sozial bedingte Assoziation handelt und nicht um einen Wesenszusammenhang. Daher kann ihre heutige Gültigkeit nicht gerechtfertigt werden. Darüber hinaus ist Mut - wie oben gezeigt wurde - nicht unbedingt ein männlicher Charakterzug, sodass die Verbindung zwischen Männlichkeit und Mut auch auf historischen, kulturellen und sozialen Faktoren gründet und mit spezifischen Bildern davon operiert, was wir für mutig halten.

In den drei vorherigen Abschnitten wurde ausgehend von Bourdieu gezeigt, dass das Nachdenken über Männlichkeit immer innerhalb des Rahmens tradierter und verinnerlichter Strukturen stattfindet. Diese Strukturen spiegeln oft eine bestimmte Ordnung der Geschlechter wider. Sich davon zu befreien, verlangt eine Reflexion über die eigenen Wahrnehmungs-, Denk-, Handlungs- und Gefühlsmuster, die unseren Weltzugang bestimmen. Einteilungen, Klassifikationen, Taxonomien, Charaktermerkmale usw., die ,in der Natur der Dinge‘ zu liegen scheinen, sind oft von sozialen Praxen abhängig. Die phänomenologischen Thesen über den Weltbezug von Individuen und Kollektiven und über die Universalität der Gefühle und ihre soziale Bedingtheit sind ein weiteres hilfreiches Werkzeug, um diese Reflexion voranzubringen. Ausgehend von Bourdieu und unter Rekurs auf phänomenologische Überlegungen konnte gezeigt werden: Männlichkeit ist das Resultat einer gesellschaftlichen Konstruktionsarbeit, in dem sich die Hierarchie der Geschlechter widerspiegelt. Da Männlichkeit eine sozial konstruierte Kategorie ist, ist keines der Merkmale, das sie in einem bestimmten Moment kennzeichnet, für sie wesentlich. Eine essenzialistische Definition von Männlichkeit, die auf der Angabe von notwendigen und hinreichenden Bedingungen beruht, ist diesen Analysen zufolge nicht möglich.

\section{MÄnNLICHKEIT ALS OffEne KATEGoRIE}

Da es keine Merkmale gibt, die als wesentliche Kriterien für eine Definition von „Männlichkeit“ einzuführen wären, und das, was wir darunter verstehen, Ergebnis einer sozialen Konstruktion ist, die eine Form von symbolischer Herrschaft zwischen den Geschlechtern und innerhalb eines bestimmten Geschlechtes impliziert, verlangt eine Annäherung an den Begriff stets eine Reflexion über gesellschaftlichen Wandel, über die verschiedene Männlichkeitsvorstellungen, die damit einhergehen und schließlich über die Machtverhältnisse zwischen den Geschlechtern. Dieses Verständnis von Männlichkeit erweckt den Eindruck, dass

38 Ebd., S. 91. 
wir es mit einer Art „leerer Kategorie“ zu tun haben: Ein Begriff, der so wandlungsfähig wäre, dass es sich nicht mehr lohnt, ihn weiter zu verwenden. Zwei Fragen kommen vor diesem Hintergrund auf: Inwiefern ist die These, dass Männlichkeit das Resultat einer Konstruktionsarbeit ist, mit der Suche nach einer Definition zu vereinbaren? Sind alle essenzialistischen Bestimmungsversuche zum Scheitern verurteilt?

Ich beginne mit der ersten Frage. Wenn wir versuchen, den Begriff der Männlichkeit anhand notwendiger und hinreichender Bedingungen zu definieren, werden wir immer wieder mit der Tatsache konfrontiert, dass viele dieser Kriterien kontingent sind und von einer bestimmten sozialen Ordnung abgeleitet werden. Wenn sich diese soziale Ordnung änderte, gälten vielleicht andere Kriterien als definitorisch für diesen Begriff. Eine Definition im klassischen Sinne der Angabe fester Kriterien scheint daher weder möglich noch wünschenswert zu sein. Dies aber impliziert nicht, dass wir auf eine Definition des Begriffs verzichten müssten. Der Begriff ist in verschiedenen akademischen und nichtakademischen Diskursen verbreitet, und er benötigt eine fundierte Theoretisierung. Der Vorschlag, den ich hier machen möchte, besteht darin, eine anti-essenzialistische Definition zu versuchen und den Begriff als „offen“ zu betrachten. Die Idee, dass es Begriffe gibt, die offen sind, aber deswegen nicht als undefinierbar betrachtet werden sollen, übernehme ich von Morris Weitz. Er schreibt: „[S]ome concepts may be and are governed by less-than-definitive sets of criteria. ${ }^{39}$ Ein Begriff ist offen, wenn er aufgrund seiner Diskutabilität, Flexibilität oder Vagheit unterschiedliche und wandelbare Bedeutungen haben kann und keines der Kriterien, die angegeben werden können, notwendig oder hinreichend ist. ${ }^{40}$ Weitz' Theorie der offenen Begriffe lässt sich von Wittgensteins Theorie der Familienähnlichkeiten inspirieren. Bei manchen Begriffen - wie etwa dem des Spiels - lassen sich keine gemeinsamen Eigenschaften finden, sondern nur Stränge von Ähnlichkeiten. Wie lässt sich diese Idee auf den Begriff der Männlichkeit anwenden?

In der Philosophie scheint es zunächst nahe zu liegen, solche schwer bestimmbaren Begriffe wie Männlichkeit durch Wittgensteins Konzept der Familienähnlichkeiten aufzuklären. Es gäbe dann verschiedene Phänomene, die wir als Männlichkeit bezeichnen, und die wir aufgrund ihrer Ähnlichkeiten zu einem Bündel zusammenbringen können. Einen Vorschlag in dieser Richtung haben z.B. Connell und Messerschmidt gemacht. Sie behaupten, dass es möglich sei, dass es auf lokaler Ebene unterschiedliche hegemoniale Herrschaften gäbe, die

39 Weitz, Morris: The Opening Mind. A Philosophical Study of Humanistic Concepts, Chicago 1977, S 23.

40 Vgl. ebd., S. X und S. 31. 
im Sinne Wittgensteins als Familienähnlichkeitsbegriffe fungierten. ${ }^{41}$ Dieser Versuch berücksichtigt zwar die Wandelbarkeit des Begriffs, doch liegt seine Schwierigkeit im Rekurs auf den Begriff der Familienähnlichkeit. Wenn wir auch einige Ähnlichkeiten zwischen verschiedenen Phänomenen erkennen können, die wir als Männlichkeit bezeichnen, wissen wir nicht, welche dieser Ähnlichkeiten relevant für das Verständnis dieses Phänomens sein sollte. Sollten wir die Assertivität, die Aggressivität, den Mut oder keine dieser Eigenschaften als ausschlaggebend nehmen? Es ist schwierig, hier eine Antwort zu finden, weil viele dieser Eigenschaften ideologisch geprägt sind. Ferner setzt das Erkennen relevanter Ähnlichkeiten bereits voraus, dass wir ein Vorverständnis des Begriffs haben. Wenn wir aber diesen Begriff philosophisch frei von theoretischen Konstrukten erforschen wollen, sollten wir dies ohne solche Vorverständnisse tun. Aus diesen zwei Gründen ist eine Definition über Familienähnlichkeiten problematisch: Sie setzt vieles von dem voraus, was sie untersuchen will.

Eine zweite Option, für die ich hier plädieren möchte, besteht darin, den offenen Charakter des Begriffs „Männlichkeit“ im Sinne einer Clustertheorie zu interpretieren. ${ }^{42}$ Dieser Idee zufolge gäbe es eine Gruppe von Eigenschaften, die den Begriff der Männlichkeit definieren. Diese Eigenschaften aber sind nicht fest bestimmt: Sie sind historisch und sozial wandelbar, sodass neue Eigenschaften angenommen werden und andere aus der Liste herausfallen können. Für eine Clusterdefinition müssen nicht alle, sondern nur einige Kriterien der Liste zutreffen, und kein Kriterium ist an sich notwendig, wenn es auch disjunktive notwendige Kriterien gibt; d.h. einige der Kriterien müssen in einem „Cluster“ gebündelt auftreten, um den Begriff verwenden zu können. Diese Möglichkeit ist weniger ambitioniert als eine Realdefinition oder als eine Definition im Sinne von Familienähnlichkeiten. Sie erlaubt uns aber, eine minimale Bestimmung des Begriffs „Männlichkeit“ zu versuchen. Warum ist eine Clusterdefinition angemessener? Solche Definitionen sind durch ihre Realitätstreue gekennzeichnet: Es gibt verschiedene Gebrauchsarten dieses Begriffs in den heutigen Diskursen, die zu berücksichtigen sind. Darüber hinaus versuchen Clusterdefinitionen auch die soziale und historische Wandelbarkeit des Konzepts zu erfassen.

Aus der vorherigen Diskussion zweier Modelle von Männlichkeit ergeben sich interessante Kriterien, um die heutzutage zirkulierenden Bilder von Männlichkeit und ihre soziale Wandelbarkeit zu verstehen. Unter den vielen Merkma-

41 Connell/Messerschmidt: Hegemonic Masculinity, in: Gender \& Society, S. 850.

42 Inspirierend finde ich hier Berys Gauts Vorschlag, Kunst als Clusterbegriff zu verstehen. Gaut, Berys: „Kunst“ als Clusterbegriff, in: Roland Bluhm/Reinold Schmücker (Hg.), Kunst und Kunstbegriffe. Der Streit um die Grundlagen der Ästhetik, Münster 2013, S. 140-165, hier S. 143. 
len, die heute angegeben werden können, um Männlichkeit zu definieren, gäbe es einige, die sich in einer Clusterdefinition bündeln ließen, derzufolge Männlichkeit mit Assertivität und Mut zu tun hat. Diese Merkmale würden ein bestimmtes Paradigma der männlichen Herrschaft wiederspiegeln, das bislang als dominant galt. Eine andere mögliche Clusterkombination verstünde Männlichkeit als eine Konstruktion, die Attribute bezeichnet, die innerhalb einer bestimmten sozialen Ordnung Männern zugeschrieben worden sind. Diese Definition reflektierte dann eine andere Ordnung der Geschlechter, in der diese Attribute als sozial bedingt erscheinen. Nicht nur diese zwei Möglichkeiten sind denkbar. Die Liste, die Männlichkeit definiert, bezöge auch Clustermöglichkeiten ein, aus denen sich andere Verständnisse von Männlichkeit ergäben und die neue Ordnungen der Geschlechter und unter Geschlechtern wiederspiegelten. Solche Clusterdefinitionen zeigen, dass es unterschiedliche Möglichkeiten gibt, die männliche Identität zu gestalten. Diese verschiedenen Möglichkeiten müssen miteinander nicht in Verbindung stehen und untereinander auch nicht ähnlich sein: Denn sie können unterschiedliche Vorstellungen der sozialen Ordnung als Hintergrund haben. Einige diese Konstruktionen spiegeln Machtverhältnisse wider, andere hingegen können Resultat eines Versuchs sein, diese Machtverhältnisse zwischen den Geschlechtern aufzugeben.

Die Konstatierung, dass es sich um einen offenen Begriff handelt, soll uns allerdings nicht dazu bringen, die essenzialistischen Definitionen, die bislang versucht wurden, als sinnlos zu betrachten. Dieser Punkt betrifft die zweite Frage dieses Abschnitts. Essenzialistische Definitionen können in einem bestimmten Sinne sehr aufschlussreich sein, besonders wenn es darum geht, die Kriterien der Liste für eine Clusterdefinition von Männlichkeit zu bestimmen. Sie zeigen uns, welche Modelle von Männlichkeit in einem bestimmten zeitlichen, historischen und kulturellen Rahmen dominant gewesen sind. Hier können wir viel über die Diskussion von Begriffen in anderen Bereichen der Philosophie lernen. Denken wir an die Definitionen von Begriffen wie „Kunst“: Auch hier handelt es sich um einen Begriff, der nicht anhand einer festen Menge notwendiger und hinreichender Bedingungen definiert werden kann, weil das, was als Kunst gilt, vom historischen Kontext abhängig ist. Dennoch zeigen uns die immer wieder neuen Bemühungen, den Begriff zu definieren, was in einem bestimmten Moment in der Gesellschaft wichtig ist und geltend gemacht wird. Essenzialistische Definitionen sind interessant, weil sie viel über die soziale Ordnung, in der sie formuliert wurden und deren Werte offenbaren. Sie sind ein heuristisches Werkzeug dafür, über die Strukturen nachzudenken, in denen sie formuliert wurden. ${ }^{43}$

43 Für Hilfe bei der Verfassung dieses Aufsatzes möchte ich mich bei Hilge Landweer, Isabella Marcinski und Matthias Warkus bedanken. 


\section{LITERATUR}

Bourdieu, Pierre: Die männliche Herrschaft, übersetzt v. Jürgen Bolder, Frankfurt a.M. 2005.

Connell, Raewyn: Masculinities, Berkeley, California/Los Angeles 2005.

Connell, Raewyn/Messerschmidt, James W.: Hegemonic Masculinity. Rethinking the Concept, in: Gender \& Society 19/6 (2005), S. 829-859.

Ekman, Paul: Emotions Revealed. Recognizing Faces and Feelings to Improve Communication and Emotional Life, New York 2004.

Gaut, Berys: „Kunst“ als Clusterbegriff, in: Roland Bluhm/Reinold Schmücker (Hg.), Kunst und Kunstbegriffe. Der Streit um die Grundlagen der Ästhetik, Münster 2013, S. 140-165.

Guthoff, Heike: Kritik des Habitus. Zur Intersektion von Kollektivität und Geschlecht in der akademischen Philosophie, Bielefeld 2013.

Landweer, Hilge: Sozialität und Echtheit der Gefühle. Geschlechtertheoretische Perspektiven, in: Agnes Neumayr (Hg.), Kritik der Gefühle, Wien 2007, S. 63-91.

Mansfield, Harvey C.: Manliness, London/New Haven, Conn. 2006.

Newmark, Catherine: Weibliches Leiden - männliche Leidenschaften. Zum Geschlecht in älteren Affektenlehren, in: Feministische Studien 26/1 (2008), S. 7-18.

Dies.: Vernünftige Gefühle? Männliche Rationalität und Emotionalität von der frühneuzeitlichen Moralphilosophie bis zum bürgerlichen Zeitalter, in: Manuel Borutta/Nina Verheyen (Hg.), Die Präsenz der Gefühle. Männlichkeit und Emotion in der Moderne, Bielefeld 2010, S. 41-55.

Nussbaum, Martha C.: „Man Overboard“, in: The New Republic vom 28.6.2006, S. $31 f$.

Ortega y Gasset, José: Über den Ausdruck als kosmisches Phänomen, übersetzt v. Helene Weyl und Ulrich Weber, in: ders., Gesammelte Werke, Band 1, Stuttgart 1954, S. 393-415.

Ders.: Über die Liebe. Meditationen, übersetzt v. Helene Weyl, Stuttgart 1957.

Scheler, Max: Über Scham und Schamgefühl, in: ders., Gesammelte Werke, Band X, hrsg. v. Maria Scheler, Bern 1957, S. 67-154.

Ders.: Erkenntnis und Arbeit, in: ders., Gesammelte Werke, Band VIII, hrsg. v. Maria Scheler, Bern 1960, S. 191-382.

Ders.: Wesen und Formen der Sympathie, in: ders., Gesammelte Werke, Band VII, hrsg. v. Manfred S. Frings, Bern/München 1973, S. 9-258.

Ders.: Ordo Amoris, in: ders., Gesammelte Werke, Band X, hrsg. v. Manfred S. Frings, Bonn 1986, S. 345-376. 
Scholz, Sylka: Männlichkeitssoziologie, Münster 2015.

Sztompka, Piotr: The Sociology of Social Change, Oxford, UK/Cambridge, Mass. 1993.

Weitz, Morris: The Opening Mind. A Philosophical Study of Humanistic Concepts, Chicago 1977.

\section{OnLine-QuelLen}

Titunik, Regina: Undermanned, in: H-Minerva, April 2007, S.1-3, auf: https://networks.h-net.org/node/5376/reviews/37050/titunik-mansfieldmanliness 